\title{
Dental Injuries Following Segmented Le Fort I Osteotomy - A Retrospective Radiographic Study of 101 Patients
}

\author{
Mawlood G1,2*, Wikström JM ${ }^{1,2}$, Lund $\mathrm{H}^{1}$ and Rasmusson $\mathrm{L}^{2}$ \\ 'Department of Oral \& Maxillofacial Radiology, Institute of Odontology, The Sahlgrenska Academy, University of Gothenburg, Gothenburg, Sweden \\ 2Department of Oral and Maxillofacial Surgery, Institute of Odontology, The Sahlgrenska Academy, University of Gothenburg, Gothenburg, Sweden
}

*Corresponding author: Goran Mawlood DDS, Department of Oral and Maxillofacial Surgery, Institute of Odontology, The Sahlgrenska Academy, University of Gothenburg, P.O. Box 450, SE 40530 Gothenburg, Sweden; Fax: +31 7863207; Tel: +46704620908; E-mail: goranmawlood@live.com

Received: December 24, 2018; Accepted: December 31, 2018; Published: January 2, 2019;

\begin{abstract}
Segmented Le Fort I osteotomy is a surgical procedure which allows the posterior segments to be repositioned more coronally to close an open bite, as well as correcting transverse discrepancies. The aim of this retrospective study was to assess the prevalence of injuries to teeth and surrounding hard tissues in patients who had undergone the Le Fort I osteotomy procedure with maxillary segmentation. In total, 101 patients were included according to predetermined criteria. Radiographs from the 6-month postoperative follow-up were reviewed in all patients. Complications such as root fractures, root resorptions, periodontal defects, sclerotic and osteolytic processes were noted. Intraoral periapical radiographs were primarily reviewed and where unavailable, panoramic radiographs were instead used. In addition, preoperative and postoperative radiographs up to 30 months were reviewed in patients with found radiographic changes at 6 months. In cases with root fracture, the medical record was reviewed in search of any additional treatment. Seven root fractures were noted in total. Only one tooth required replacement, in this case with a dental implant. Clinically significant complications to teeth adjacent to vertical osteotomies from the Le Fort I osteotomy with maxillary segmentation were uncommon.
\end{abstract}

Keywords: Complications; Interdental Osteotomy; Le Fort I Osteotomy; Maxillary Segmentation; Orthognathic Surgery

\section{Introduction}

Transverse discrepancies and anterior open bite can be successfully treated with a combination of fixed orthodontic appliances and maxillary segmentation $[1,2]$. If an open bite exists on a level occlusal plane, it can be surgically corrected in one piece by reducing the height of the maxilla, mostly in the posterior portion, after a one piece Le Fort I osteotomy. A step in the occlusal plane, however, necessitates segmentation of the maxilla for proper correction of the discrepancy [3]. The segmented Le Fort I osteotomy is a surgical procedure which allows the surgeon to reposition each segment into the desired positions so that a narrow maxilla can be widened or to close an open bite. In such operations, vertical interdental osteotomies are performed in order to segment the maxilla. There are to our knowledge relatively few studies reporting on complications to teeth and surrounding bone tissue in conjunction with vertical interdental osteotomies. Kahnberg and colleagues reported a low incidence of hard tissue complications and iatrogenic damage to teeth [4] while another study by Schultes et al. indicaes that interdental osteotomies may result in severe periodontal tissue breakdown and segmental loss of teeth [5]. Schou et al. and Morgan and Fridrich did not find that interdental osteotomies lead to significant marginal bone destruction $[6,7]$ which was later confirmed in two studies published more recently $[8,9]$. The aim of this retrospective study was to assess the prevalence of injuries to teeth and surrounding hard tissues following the segmented Le Fort I osteotomy.

\section{Materials and Methods}

Patients subjected to segmented Le Fort I osteotomy between January 2005 and December 2015 at the Department of Oral and Maxillofacial Surgery, The Sahlgrenska Academy, University of Gothenburg were included in the study according to following inclusion and exclusion criteria:

\section{Inclusion criteria}

- Undergone segmented Le Fort I osteotomy with or without simultaneous mandibular surgery between January 2005 and December 2015.

- Combined orthodontic and surgical treatment.

- Available intra-oral and/or panoramic radiographs 6 months postoperatively.

\section{Exclusion criteria}

- Undergone segmented Le Fort I osteotomy without preoperative orthodontic treatment.

- No available intra-oral or panoramic radiographs 6 months postoperatively.

- Undergone the operation more than once. 
A total of 101 patients (mean age 22.8 years, median age 21 years, range 17-61 years, $\mathrm{SD}= \pm 7.56$ ) were included (Table 1 ). The most common diagnosis necessitating maxillary segmentation was an anterior open bite, which meant the maxilla was typically segmented into four pieces. Radiographs were analysed under dimmed lighting on computer monitors (HP Elite Display E222, 1920x1080) by two authors (G.M and J.M.W). When agreement between authors was not reached, the radiographs in question were presented to an oral and maxillofacial radiologist (H.L) for decision. Primarily, intraoral periapical radiographs were examined and secondarily panoramic radiographs. Radiographs for all 101 patients were analysed at 6 months after surgery with respect to teeth and surrounding tissues adjacent to the interdental osteotomies. The following radiographic changes were noted: osteolytic processes (i.e. widened periodontal ligament spaces (WPLs) and periapical osteolyses), sclerotic processes, marginal bone loss, angular bony defects, root resorptions and root fractures. The preoperative and all follow up radiographs up to 30 months postoperatively were analysed in cases with found radiographic changes at radiographs taken at the 6-month recall.

Table 1. Collected data at the 6-month recall.

\begin{tabular}{|l|c|}
\hline Sex \\
\hline Male & 60 \\
\hline Female & 41 \\
\hline Radiographs & 42 \\
\hline Intra-oral and panoramic & 59 \\
\hline Panoramic only & \\
\hline Interdental osteotomies & 10 \\
\hline Unilateral & 91 \\
\hline Bilateral & \\
\hline
\end{tabular}

\section{Surgical procedure}

The surgical procedure was carried out as follows $[1,10]$ : Incisions were made in the buccal sulcus, extending from the second premolar to the inferior aspect of the nasal spine. The periosteum was then elevated at the superior aspect of the incision, extending posteriorly to expose the lateral wall of the maxillary sinus. Nasal mucosa was then elevated from the piriform aperture and osteotomies were then carried out, beginning with a cut extending from the piriform rim to the zygomatic buttress and posteriorly through the lateral wall of the maxillary sinus. After sectioning the lateral nasal wall, the maxilla was then disengaged from the pterygoid bone using an osteotome inferior to the pterygomaxillary cleft. The lateral wall of the sinus was then separated using an osteotome in a posterior direction. At this point in the operation, maxillary segmentation was carried out. This was done by elevating the periosteum in the region of the inter dental osteotomy, most commonly between the canine and the first premolar bilaterally. The buccal cortical bone was cut with a bur and the osteotomy was then completed with an osteotome. The maxilla was at this stage down fractured with inferior pressure on the anterior teeth. Complete mobilization of the maxilla was obtained with an osteotome applying force in an anterior direction from the maxillary tuberosity. The maxillary segmentation was completed proceeding from the superior aspect of the maxilla. Stainless steel wires were used to fixate the maxilla to the mandible, with a surgical splint for guidance. The maxillomandibular complex was then fixated with titanium plates and screws in the desired position. Soft tissue was closed beginning with appropriate positioning of the facial muscles, concluding with closure of the mucosa.

\section{Statistical analysis}

The Two-Proportion Z-Test (SPSS Inc., Chicago, Illinois, USA) was utilised in order to assess the correlation of the proportion of the radiographic changes with the type of radiograph analysed and also comparing unilateral with bilateral osteotomies. $\mathrm{p}<0.05$ was considered statistically significant.

\section{Results}

The results are summarised in (Table 2). Ninety-one patients underwent bilateral interdental osteotomies and 11 patients unilateral. 384 teeth were therefore examined at the 6-month followup. All 35 patients with radiographic changes at 6 months had preoperative radiographs available, one of which underwent the unilateral osteotomy, meaning 138 teeth were examined at this point. At the 18-month recall, radiographs for 25 patients were available and 98 teeth were included. At 30 months, only one patient had radiographs available, meaning the inclusion of 4 teeth. Overall, $35 \%$ of patients were found to have some form of radiographic changes at the 6-month recall. Male and female patients had an equal likelihood of having radiographic changes at 6 months. Fifty percent of patients with intraoral radiographs were found to have radiographic changes and $24 \%$ in the group with only panoramic radiographs available. This difference was statistically significant $(\mathrm{z}=2.7, \mathrm{p}<0.01)$. Ten percent of patients with unilateral osteotomies and $37 \%$ of patients with bilateral osteotomies exhibited radiographic changes, but this difference was not statistically significant $(\mathrm{z}=1.7, \mathrm{p}>0.05)$.

Table 2. Summary of radiographic changes.

\begin{tabular}{|l|l|l|l|l|}
\hline & Preop. & $\mathbf{+ 6}$ months & $+\mathbf{1 8}$ months & $+\mathbf{3 0}$ months \\
\cline { 2 - 6 } & $(\mathbf{n}=\mathbf{1 3 8})$ & $\mathbf{( n = 3 8 4 )}$ & $(\mathbf{n}=\mathbf{9 8})$ & $\mathbf{( n = 4 )}$ \\
\hline WPL & 7 & 4 & 1 & 0 \\
\hline Periapical osteolysis & 3 & 5 & 2 & 0 \\
\hline Sclerotic process & 2 & 5 & 1 & 0 \\
\hline Marginal bone loss & 1 & 3 & 2 & 0 \\
\hline Angular bony defect & 0 & 7 & 4 & 0 \\
\hline Root resorption & 20 & 29 & 21 & 0 \\
\hline Root fracture & 0 & 7 & 2 & 1 \\
\hline Total defects & 33 & 60 & 33 & 1 \\
\hline WPL: widened periodontal ligament space & \\
\hline
\end{tabular}

\section{Osteolytic processes}

The osteolytic processes registered were periapical radiolucencies and widened periodontal ligament spaces (WPLs). At the initial 6-month follow-up, 4 of teeth adjacent to the osteotomies were found to have WPLs, two of which were seen on the preoperative 
radiographs. The remaining two teeth had no visible WPLs at 18 months. However, an additional WPL was found on a different tooth at this point. Five teeth were found exhibiting periapical osteolytic processes at the 6-month mark, one of which was present preoperatively. At the 18-month follow-up, only two of the patients (two of the teeth) had available radiographs, where one tooth exhibited a remaining periapical radiolucency. Another of the patients, with an affected tooth, who lacked 18-month radiographs did have a 30-month followup, however, where the periapical radiolucency was no longer visible.

\section{Sclerotic processes}

Five teeth were found to have sclerotic processes at 6 months, two of which were visible preoperatively. At the 18 -month recall, one of the teeth with postoperative sclerosis lacked radiographs and in the remaining two, the scleroses were no longer visible.

\section{Marginal bone loss and angular bony defects}

Three osteotomy sites were found to have a marginal bone level located $\geq 3 \mathrm{~mm}$ from the CEJ 6 months postoperatively, two of these sites had a reduced marginal bone level compared with radio- graphs taken preoperatively. At 6 months, the marginal bone level at these two sites was measured at $7 \mathrm{~mm}$ and $4.5 \mathrm{~mm}$ respectively, compared to $1.5 \mathrm{~mm}$ and $2 \mathrm{~mm}$ at radiographs taken before surgery. Two of these patients were examined with panoramic radiographs at 18 months and no further bone loss could be detected. The third patient, with marginal bone loss of $2.5 \mathrm{~mm}$, did not have available radiographs at 18 months. Seven interproximal tooth surfaces were found exhibiting adjacent angular bony defects at 6 months, none of which were visible on the preoperative radiographs. At the 18-month followup, one of the bony defects lacked radiographs, and of the remaining 6 , two were no longer present.

\section{Root resorptions}

Twenty-nine teeth were found with root resorptions at 6 months, 20 of which were present preoperatively, indicating that 9 had arisen after surgery. Two of these teeth lacked radiographs at 18 months, and an additional 6 teeth were registered as having root resorptions that were not noted in earlier radiographs.

\section{Root fractures}

Seven teeth (5 premolars and 2 canines) were found with root fractures at 6 months, none of which were present preoperatively (Figure 1). At 18 months, three of these teeth lacked radiographs and in one other case the intraoral radiograph did not capture the area with the root fracture. Of the three remaining teeth examined at 18 months, one was still visible and had healed into the bone, one was examined only with panoramic imaging and was no longer discernible. The third tooth was eventually extracted and replaced with an implant. There was a 30-month follow-up for one of the fractures not examined at 18 months, where it could still be seen to be healed into the bone.

\section{Discussion}

The aim of this study was to assess the prevalence of injuries to teeth and surrounding hard tissues adjacent to vertical interdental osteotomies in patients who had undergone the Le Fort I osteotomy with maxillary segmentation. The main drawback of this study was the relatively small proportion of intraoral radiographs covering the interdental osteotomy area. A majority of the patients had only panoramic radiographs available, hindering the detection of the subtle radiographic changes which were the subject of this study. There was significantly fewer radiographic changes found on panoramic radiographs $(\mathrm{z}=2.7, \mathrm{p}<0.01)$. Intraoral radiographs are more appropriate in the detection of these radiographic changes due to their higher resolution [11]. We suggest therefore that intraoral radiographs are taken in cases where interdental osteotomies have been performed, as a complement to extraoral imaging techniques. Panoramic radiographs are taken routinely during the follow up of patients undergoing orthognathic surgery for evaluation of healing of bone segments and fixation material. Overlapping in the upper premolar region and superimposition of osteosynthesis plates was not uncommon in some of the panoramic radiographs included in this study, making it difficult to distinguish radiographic changes in these cases. According to Lofthag Hansen and co-workers, periapical lesions not detected on intraoral radiographs may be visible when using CBCT [12]. Routine use of three-dimensional imaging techniques in the follow-up of patients undergoing orthognathic surgery is however not justified, due to the relatively high radiation exposure.

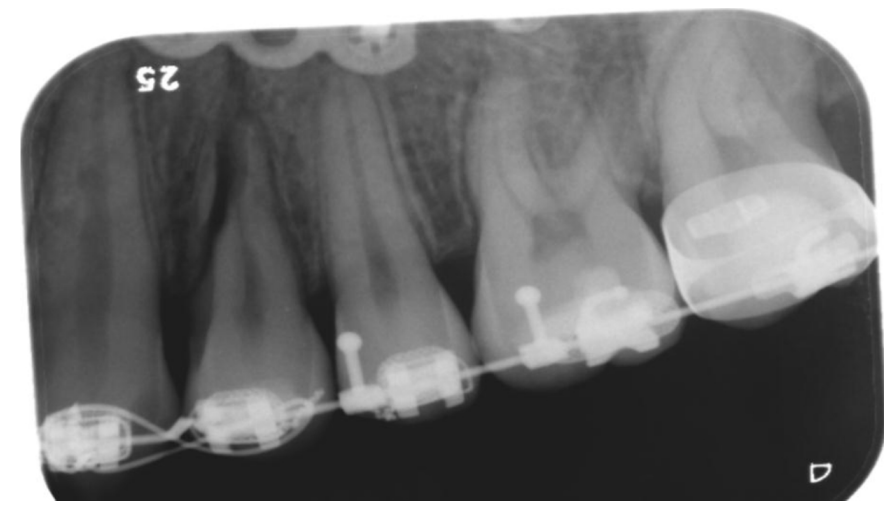

Figure 1. Periapical radiograph of a patient presenting with root fracture of 24.

To this study's advantage is the relatively large patient sample included, increasing the generalisability of the results. No differences were found between the sexes with regards to the proportion of radiographic changes found. Ten percent of patients that underwent a unilateral interdental osteotomy exhibited radiographic changes at 6 months compared to $37 \%$ in the group with bilateral osteotomies. This difference was not found to be statistically significant, perhaps owing to the small population in this study that underwent the unilateral variant. A higher prevalence in the bilateral group would be expected in that a patient is more likely to receive dental injury when two interdental osteotomies are performed compared to one.

Four teeth in this study were found to have periapical osteolytic processes not present on preoperative radiographs, three of these teeth had additional follow-up radiographs, where two of the periapical osteolyses were no longer visible, indicating that healing had occurred in these cases. It is possible that the presence of periapical osteolyses is due to the teeth being devitalised by the operation. A study by Bell 
showed that horizontal osteotomy cuts within $5 \mathrm{~mm}$ of the apices of adjacent teeth could disturb pulpal blood circulation and thus risk devitalising the teeth [13]. In the present study however, the horizontal osteotomies were seen radiographically to be of adequate distance from the apices. Another possibility is that vertical osteotomies in close proximity to the apex of the adjacent teeth have disturbed the blood circulation in a similar manner. Nevertheless, none of the affected teeth underwent endodontic treatment within the confines of this study. Kahnberg et al. proposed that endodontic treatment should be considered in cases where the tooth is symptomatic. They also recommend regular clinical follow-up in cases where a periapical osteolysis is radiographically visible [4].

In this patient material, two percent (7 of 384) of the teeth were damaged during the vertical interdental osteotomy procedure, resulting in root fractures, one of which was subsequently replaced by an implant. The root fracture that was replaced with an implant was due to the fracture line reaching the level of the marginal bone, thwarting healing into the bone. Horizontal root fractures located within the coronal third of the root are associated with poor prognosis [14]. Orthodontic tipping of the roots of adjacent teeth away from the planned vertical osteotomy site is important to reduce the risk of iatrogenic root fracture [10] and could perhaps also serve to reduce the risk of compromised pulpal blood flow. Our results regarding postoperative loss of teeth are in line with what was reported by Kahnberg et al., Ho et al. and Rodrigues et al. [4, 8, 9]. Schultes et al. reported a higher incidence of postoperative tooth loss, likely due to the high prevalence of periodontal injury in that study [5]. Root resorption was seen in 29 teeth at the 6 month follow up, 20 of which were visible preoperatively. These findings imply that the vast majority of root resorptions in this study were due to the orthodontic pretreatment $[15,16]$. In a study utilising $\mathrm{CBCT}$, it has been shown that root resorptions can be detected in varying degrees in almost all teeth following treatment with fixed orthodontic appliances [17]. Root resorptions that were detected postoperatively may be influenced by surgical trauma and/or orthodontic treatment. Marginal bone loss was detected in two osteotomy sites which is comparable to what is reported by Schou et al. and Kahnberg et al. [4, 6]. Small, subclinical alterations in marginal bone level were however not registered in this study. One limitation of intraoral and panoramic radiographs in assessing the marginal bone level is that measurements can only be performed at the interproximal sites. Orthodontic forces have been shown to lead to a significant reduction of the crestal height at the buccal, lingual and palatal aspects of teeth. [18].

Radiographic changes present on preoperative radiographs indicate a non-surgical etiology. When taking this into account, the proportion of patients with radiographic changes falls from $35 \%$ to $28 \%$. On the level of individual teeth adjacent to the osteotomy sites, the corresponding risk for a tooth adjacent to the osteotomy site would be $9 \%$ in this study, as 34 teeth exhibited radiographic changes at the 6-month follow-up which were not present preoperatively. However, had patients with only panoramic radiographs available at 6 months been excluded from this study, due to the aforementioned lower resolution of panoramic radiographs, the risk of incurring radiographic changes to an individual tooth adjacent to the vertical osteotomy could be said to be $15 \%$ (excluding findings present preoperatively).

\section{Conclusion}

Overall it can be concluded from this study that the risk posed to teeth adjacent to vertical osteotomies in conjunction with Le Fort I osteotomies is low, with 1 in 384 teeth included in this study requiring extraction and replacement.

Conflict of interest: We have no conflicts of interest to disclose.

Ethical approval: Ethical approval was not required.

\section{References}

1. Kahnberg KE (2007) Transverse expansion of the maxilla using a multisegmentation technique. Scand J Plast Reconstr Surg Hand Surg 41: 103-108.

2. Silva I, Suska F, Cardemil C, Rasmusson L (2013) Stability after maxillary segmentation for correction of anterior open bite: a cohort study of 33 cases. $J$ Craniomaxillofac Surg 41: e154-158. [crossref]

3. Hupp JR, Ellis E, Tucker MR (2014) Contemporary Oral and Maxillofacial Surgery. St Louis MO: Elsevier Pg No: 520-539.

4. Kahnberg KE, Vannas-Löfqvist L, Zellin G (2005) Complications Associated with Segmentation Of The Maxilla: A Retrospective Radiographic Follow Up Of 82 Patients. International Journal of Oral and Maxillofacial Surgery 34: 840-845.

5. Schultes G, Gaggl A, Kärcher H (1998) Periodontal disease associated with interdental osteotomies after orthognathic surgery. J Oral Maxillofac Surg 56: 414-417. [crossref]

6. Schou S, Vedtofte P, Nattestad A, Stoltze K (1997) Marginal bone level after LeFort I osteotomy. British Journal of Oral and Maxillofacial Surgery 35: 153-156.

7. Morgan TA, Fridrich KL (2001) Effects of the multiple-piece maxillary osteotomy on the periodontium. Int J Adult Orthodon Orthognath Surg 16: 255.

8. Ho MW, Boyle MA, Cooper JC, Dodd MD, Richardson D (2011) Surgical complications of segmental Le Fort I osteotomy. Br J Oral Maxillofac Surg 49: 562-566. [crossref]

9. Rodrigues DB, Campos PSF, Wolford LM, Ignácio J, Gonçalves JR (2018) Maxillary Interdental Osteotomies Have Low Morbidity for Alveolar Crestal Bone and Adjacent Teeth: A Cone Beam Computed Tomography-Based Study. J Oral Maxillofac Surg S0278-2391: 30111-30113.

10. Andersson L, Kahnberg KE, Pogrel MA (2010) Oral and Maxillofacial Surgery 2010; Chichester: John Wiley \& Sons Ltd Pg No: 1009-1012.

11. White S, Pharoah M (2014) Oral radiology 2014; St. Louis, Missouri: Elsevier Pg No: 177

12. Lofthag-Hansen S, Huumonen S, Gröndahl K, Gröndahl HG (2007) Limited conebeam CT and intraoral radiography for the diagnosis of periapical pathology. Oral Surg Oral Med Oral Pathol Oral Radiol Endod 103: 114-119. [crossref]

13. Bell WH (1969) Revascularization and bone healing after anterior maxillary osteotomy. A study using adult rhesus monkeys. Journal of Oral Surgery 27: 249.

14. Cvek M, Andreasen JO, Borum MK (2001) Healing of 208 intra-alveolar root fractures in patients aged 7-17 years. Dent Traumatol 17: 53-62. [crossref]

15. Goldson L, Henrikson CO (1975) Root resorption during Begg treatment; a longitudinal roentgenologic study. Am J Orthod 68: 55-66. [crossref]

16. Linge L, Linge B (1991) Patient characteristics and treatment variables associated with apical root resorption during orthodontic treatment. American Journal of Orthodontics and Dentofacial Orthopedics 99: 35-43.

17. Lund H, Gröndahl K, Gröndahl HG (2012) Cone beam computed tomography evaluations of marginal alveolar bone before and after orthodontic treatment combined with premolar extractions. Eur J Oral Sci 120: 201-211. [crossref]

18. Lund H, Gröndahl K, Hansen K, Gröndahl HG (2012) Apical root resorption during orthodontic treatment. A prospective study using cone beam CT. Angle Orthod 82: 480-487. [crossref]

Citation:

Mawlood G, Wikström JM, Lund H, Rasmusson L (2019) Dental Injuries Following Segmented Le Fort I Osteotomy - A Retrospective Radiographic Study of 101 Patients. J Dent Maxillofacial Res Volume 1(2): 1-4. 